for overcoming quantum challenges, and developed effective plans to attract and engage students and young researchers to produce next-generation researchers in quantum science and engineering," says Kono of their effort to secure a QLCI grant.

Both Kono and $\mathrm{Fu}$ recognize the important role of materials research in advancing quantum science, and both the Texas Quantum Institute and Institute for Hybrid Quantum Systems include materials researchers. Kono characterizes quantum materials as being an "essential underpinning” of future QIS technologies, and Fu says that "it is well understood that advancements in materials will need to be made to enable scalable quantum information technologies."

Producing materials of higher purity, increasing control over materials synthesis to produce desirable properties for quantum information, and developing new and more sensitive means of measuring materials properties are issues that, according to $\mathrm{Fu}$, must be addressed by materials scientists to enable the QIS technologies of the future. In addition,
$\mathrm{Fu}$ points to the potential use of quantum simulation to accelerate understanding and discovery of materials and says, "QIS and materials are profoundly related."

"This is a very exciting time for quantum information science," says Fu because not only has the US government recognized the importance of QIS, but it is also the "first time we are seeing a really significant investment into quantum technologies from industry." The institute structure that is part of the QLCI program will help further encourage industry investment and participation because it creates an environment where multidisciplinary teams from academia, government, and the private sector will need to come together to focus on large-scale challenges.
"A collection of individual investigators could never have the sustained, cross-disciplinary, holistic effort needed" to address the sizable challenges in both accelerating QIS advancements and developing the quantum information scientists and engineers of the future, Kono says. But Kono, Fu, and a host of other researchers are already utilizing the QLCI program to convene the teams and build the environment necessary to push the frontiers of QIS.

Jennifer A. Nekuda Malik

\section{EU companies increased R\&D investment in 2019}

$\mathrm{C}$ ompanies in the EU have increased their investment in R\&D for the ninth consecutive year, but levels remain below the global growth rate. In the 2018-2019 time frame, EU companies invested $4.7 \%$ more in R\&D. This growth is driven by the automobile, health, and information and communication technology (ICT) sectors.

In the face of an ever-increasing global technological race, the top 2500 industrial players worldwide increased their R\&D investment by $8.9 \%$ in 2018-2019. EU companies account for 17 of the top 50 corporate R\&D investors. The growth in investment by EU-based companies is, however, outpaced by their US and Chinese counterparts: the former increased their $\mathrm{R} \& \mathrm{D}$ investment in that time frame by $10.3 \%$, the latter by $26.7 \%$.
These are some of the main findings of the 2019 EU Industrial R\&D Investment Scoreboard (doi:10.2760/04570), published by the European Commission in December of last year. It provides an annual in-depth analysis of the most recent investment trends of the worlds' leading industrial R\&D players contributing to understanding the positioning of the EU companies in the global landscape.

Mariya Gabriel, EU Commissioner for Innovation, Research, Culture, Education, and Youth, says, "Companies based in the EU keep increasing their R\&D investments, but much more is needed in the global race for technological leadership in deep-tech and sustainability. We will launch a fully fledged European Innovation Council to invest in high-risk companies creating new markets. If we want Europe to drive the transition to a climate-neutral economy, we need to redouble our efforts."

EU-based automotive companies have contributed most to R\&D growth in the EU and prepare for the future with a broad patent portfolio to reduce vehicle emissions (behind Japan) and for autonomous vehicles. With aims to meet the UN sustainable development goals (SDGs), the report published, "Toyota had the most green patents but the top 25 global companies by number of green patents comprise EU firms such as Bosch, Volkswagen, Airbus and Rolls-Royce." Furthermore, to meet the SDGs, industrial R\&D contributed to technologies such as artificial intelligence, biotechnology, nanotechnology, graphene, and improved clean energy generation and storage technologies.

The EU Industrial R\&D Investment Scoreboard has been published annually since 2004 by the European Commission's Directorate-General for Research and Innovation and the Joint Research Centre. 